\title{
La expresión de las emociones en la Comunicación Virtual: El Ciberhabla
}

\section{Emotions expression in Virtual Communication: NetSpeak}

\author{
Isabel Cuadrado Gordillo \\ Profesora del Departamento de Psicología y Antropología \\ (Universidad de Extremadura) \\ Guadalupe Martín-Mora Parra \\ Doctoranda (Universidad de Extremadura) \\ Inmaculada Fernández Antelo \\ Profesora Departamento de Ciencias de la Educación, \\ Área Didáctica y Organización Escolar \\ (Universidad de Extremadura)
}

Fecha de recepción: 16 de abril de 2014

Fecha de revisión: 10 de enero de 2015

Para citar este artículo: Cuadrado Gordillo, I., Martín-Mora Parra, G. y

Fernández Antelo, I. (2015): La expresión de las emociones en la Comunicación Virtual: El Ciberhabla, Icono 14, volumen (13), pp. 180-207. doi: 10.7195/ri14.v13i1.716 
181 | Isabel Cuadrado, Guadalupe Martín-Mora e Inmaculada Fernández

\section{Resumen}

La comunicación mediada por ordenador se ha convertido en una de las principales herramientas de comunicación de la sociedad. La proliferación en Internet de foros y redes sociales, ha modificado los intereses y usos del tiempo libre, especialmente entre adolescentes y jóvenes. Este tipo de comunicación comparte con la escritura y con la interacción oral, algunas de sus características, sin embargo, sus peculiaridades han dado lugar a la aparición de otras nuevas. Entre estas últimas, destacan los emoticonos. En los últimos tiempos su número y complejidad ha aumentado significativamente, al igual que sus funciones.

El presente estudio pretende hacer un análisis extenso del uso y las funciones con las que personas de distintas edades emplean los emoticonos en el contexto de la Comunicación Mediada por Ordenador. Para ello se ha analizado una muestra de mensajes extraídos al azar de seis foros de discusión asincrónica distintos con una metodología interpretativo-descriptiva.

\section{Palabras clave}

Comunicación Mediada por Ordenador - Comunicación No Verbal - Internet - Redes Sociales - Emoticonos

\section{Abstract}

Computer mediated communication is a new kind of communication that has become in one of the main characteristic of our society. Millions of people around the world use social networks to improve their opportunities to satisfy their communicational needs, especially adolescence and young people. The increase of this kind of communication has modified people interests and how they spend their free time. Computer mediated communication and traditional communication has too many characteristics in common, but it is important to say that there are other ones that are different too, for example emoticons use.

This study intends to do a comprehensive study about the use and function of emoticons that people from different ages do in Computer Mediated Communication.

ICONO14 | Año 2015 Volumen $13 N^{\circ} 1$ | ISSN: 1697-8293 | DOI: ri14.v13i1.716 
For this reason, a sample of messages extracted of six different asynchronous forums has been analyzed with an interpretative-descriptive methodology.

\section{Key Words}

Computer Mediated Communication - Non Verbal Communication - Internet - Social Networks - Emoticons

\section{Introducción}

La clasificación tradicional que se hace del lenguaje incluye aspectos verbales y no verbales. Los aspectos verbales son comúnmente conocidos por todas las personas, estudiados de manera formal en los centros educativos y utilizados diariamente tanto en la lectura, como en la escritura y la comunicación cara a cara. Sin embargo, a menudo se olvida que los aspectos no verbales transmiten información igualmente importante en las conversaciones cara a cara. La comunicación no verbal va más allá de las palabras y aporta aspectos tales como los sentimientos y el estado de ánimo de las personas.

La evolución tecnológica y la expansión en el uso de Internet, ha dado lugar a un nuevo tipo de comunicación, la comunicación mediada por ordenador; un nuevo tipo de espacio comunicativo, el ciberespacio; y un nuevo tipo de lenguaje que, a priori, no cuenta con los recursos necesarios para transmitir la información no verbal. A pesar de ello, la posibilidad de comunicarse con otras personas de manera virtual ha hecho que la vertiente más social de Internet haya crecido gracias a la aparición de herramientas tales como el correo electrónico y, más recientemente el chat, los foros de discusión, la mensajería instantánea (Moral, 2008) y las redes sociales.

El concepto de comunicación mediada por ordenador surge en el contexto universitario a mediados de los años 80 del siglo XX para hacer referencia a una nueva modalidad comunicativa que tenía como protagonistas a los ordenadores dentro de un contexto interactivo (George \& Totsching, 2001). Agrupa como campo de investigación a un grupo de disciplinas diferentes tales como las ciencias del lenguaje, las ciencias de la información y de la comunicación, y la educación (Álvarez, 2008). Es definida por Mason (1990, p.223) como "conjunto de posibilidades que tienen lugar cuando los ordenadores y las redes de telecomunicaciones son usadas como herramientas en los procesos de comuni-

DOI: ri14.v13i1.716 | ISSN: 1697-8293 | Año 2015 Volumen 13 Nº 1 | ICONO14 
cación para componer, almacenar, transmitir y procesar la información". Recientemente, autores como Crystal (2013) han ampliado el concepto como consecuencia del avance tecnológico que ha propiciado la aparición de dispositivos (como el teléfono móvil inteligente o Smart Phone) que permiten el intercambio de mensajes virtuales. De este modo, se empieza a hablar de comunicación mediada electrónicamente.

En relación con las posibilidades comunicativas, no cabe duda que el medio electrónico facilita a la vez que constriñe nuestra capacidad de comunicar. La actual red 2.0 es social y permite la producción, difusión, recepción e intercambio de todo tipo de archivos: escritos, de audio, video, etc. (Cebrián, 2008). Además, la ampliación del ancho de banda (Castells, Fernández-Ardévol, LinchaunQiu \& Sey, 2006), da lugar a un aumento de la velocidad en el flujo comunicativo. Como consecuencia, los usuarios de Internet dejan de ser receptores pasivos, para ser miembros activos que construyen, crean y ofrecen información a otros, sin ninguna restricción. Sin embargo, desde otro punto de vista, hay que resaltar que el medio tecnológico que permite el acceso a la Red (ordenadores de sobremesa, portátiles, Smart phones, tablets, etc.), cuenta con una serie de características implícitas que inciden en la comunicación que se da. Propiedades como los caracteres del teclado, tipo, tamaño y configuración de la pantalla, tipo de conexión... influyen en la capacidad lingüística expresiva y receptiva. Siguiendo las palabras de Crystal (2002, p. 37) "tanto el remitente como el destinatario están limitados por las propiedades del software y del hardware que les vincula". Desde este punto de vista, pueden señalarse algunas características que diferencian la comunicación mediada por ordenador de la comunicación tradicional (cara a cara) (Moral, 2002):

- Anonimato

- Distanciamiento físico

- Tiempo. La CM0 puede ser sincrónica, es decir en tiempo real, o asincrónica. El tiempo asincrónico hace que el usuario tenga minutos, horas o incluso días antes de responder al mensaje.

- Ausencia de comunicación no verbal

ICONO14 | Año 2015 Volumen 13 N 1 | ISSN: 1697-8293 | DOI: ri14.v13i1.716 
Estas propiedades dan lugar a obvias dificultades de comunicación, sin embargo, desde hace más de 25 años, los millones de usuarios que utilizan medios electrónicos, han adoptado los recursos necesarios para conseguir una transmisión eficaz de información. De hecho, aun siendo Internet un fenómeno sustentado en la palabra escrita, no puede dejar de notarse el aumento del lenguaje oral en la red a través de videos, sonidos, e imágenes (emoticonos $\mathrm{y}$, en los últimos años, gifs animados ${ }^{1}$ ). La unión de ambos tipos de características ha dado lugar a un nuevo tipo de lenguaje, que supone la unión de la escritura y la oralidad, conocido como ciberhabla (Crystal, 2002). El empleo de este lenguaje en Internet cuenta con detractores que afirman que su uso supone una perversión del lenguaje, pero también defensores que señalan como este nuevo lenguaje agrega capital social a la cultura (Valencia \& García, 2010).

La introducción de elementos no puramente lingüísticos en el ciberhabla responde, probablemente, a la necesidad humana de añadir expresividad, sentimiento y emoción a sus mensajes. Para ello se utilizan diversas técnicas (por ejemplo, repetición de letras) en un intento de emular el lenguaje hablado (Kalman y Gergle, 2014). Entre todos los recursos que se emplean en la red para transmitir información no verbal, destaca, el empleo de emoticonos.

La palabra emoticón es un neologismo que proviene de la unión de las palabras "emotive" e "icón" en inglés, emoción e icono en español (Hernández \& Miraflores, 2007). Originalmente, como casi todos los inventos, fueron creados para resolver un problema concreto. El intercambio de bromas entre los científicos de la Carnegie Mellon University a través de Arpanet (precursora de Internet) puso de manifiesto que era necesario crear una clave para distinguir si los mensajes escritos debían ser tomados o no en serio. El científico Robert E. Falhman propuso utilizar dos puntos, un guión y un paréntesis al final de los correos electrónicos. El emoticono :-) serviría para marcar e-mails informales o jocosos, mientras que :-( se utilizaría para marcar emails urgentes o serios. Además recomendaba “Leedla de lado" (Elmundo.es, 2007).

Si bien es cierto que el origen del emoticón parece remontarse a una fecha anterior (concretamente 1967, en un artículo de Reader's Digest), Falhman es señalado como el inventor debido a que fue el primero que sugirió cómo podrían aplicarse en contextos interactivos (Wei, 2012). Por tanto, su uso ha supuesto la creación de una

DOI: ri14.v13i1.716 | ISSN: 1697-8293 | Año 2015 Volumen 13 Nº 1 | ICONO14 
herramienta cuyo principal objetivo parece ser suplir las carencias de la comunicación mediada por ordenador, no solo a la hora de expresar emociones, sino también cuando se quieren hacer chistes, transmitir ironías y sarcasmos (Jibril \& Abdullah, 2013).

La utilización de emoticonos con el paso del tiempo ha evolucionado, su número ha crecido y se han diversificado, tanto en forma como en función e intención. Al principio, representaban emociones muy básicas, sin embargo, en la actualidad forman un grupo complejo y, en ocasiones ambiguo, cuya interpretación es cada vez más difícil de realizar ya que en muchas ocasiones parecen ser altamente dependientes del contexto (Vandergriff, 2013). No obstante, todos tienen un carácter comunicativo que ha dado lugar a la creación de un código entre emisor y receptor (Moral \& García, 2003).

La investigación científica sobre el uso de emoticonos en la Red comenzó a principio de los años 90 del siglo XX. Autores como Lea y Spears (1992), Markus (1994) y Herring (1996) partían de la hipótesis de que los emoticonos eran herramientas muy valiosas que podían ser usadas para compensar la ausencia de señales emotivas y sociales en las conversaciones en línea. Estos trabajos a menudo especificaban, en palabras de Lea y Spears (1992, p. 321), que en la comunicación mediada por ordenador "el significado de las marcas paralingüísticas depende del contexto individual o de grupo que está pre-establecido para la comunicación" (Baron, 2009).

Otros autores como Kavanaugh, Carroll, Rosson, Zin y Reese (2005) a través de distintos estudios, llegaron a la conclusión de que las señales paralingüísticas en la comunicación mediada por ordenador parecían ser más necesarias en los casos en los que las personas se comunicaban con extraños en lugar de con conocidos o amigos. En esta línea, Darics (2010) señaló que el uso del emoticón "carita sonriente" parece ser esencial en contextos laborales para crear un ambiente colaborativo.

Joe Walther y Kyle D'Addario en 2001 estudiaron en qué medida los internautas se basaban en el significado de los emoticonos a la hora de interpretar los mensajes que recibían. Los resultados que encontraron apuntaban a que en la comunicación en línea uno a uno, los ciberhablantes interpretaban los mensajes basándose más en el contenido verbal que en los emoticonos. Postmes, Spears, y Lea (2000) concluyeron que el uso de emoticonos es muy variable de un grupo de comunicación en línea

ICONO14 | Año 2015 Volumen $13 N^{\circ} 1$ | ISSN: 1697-8293 | DOI: ri14.v13i1.716 
a otro, y lo que es aún más importante, un mismo usuario varía la utilización que hace de ellos según el grupo en el cual esté participando. Byron y Baldridge (2007) encontraron que la personalidad era también un factor determinante en el grado en el que las señales no verbales eran incorporadas a la comunicación en línea.

Desde otro punto de vista, algunos de los estudios realizados centran su atención en las posibles diferencias de género en su utilización. Sus resultados son variables. Witmer y Katzman (1997) hallaron en un grupo de noticias que mujeres de contabilidad usaban más emoticonos que los hombres, aunque su uso general era bajo. Wolf (2000) encontró resultados parecidos, sin embargo, descubrió que el uso que hacían los hombres de los emoticonos aumentaba cuando formaban parte de grupos mixtos. Por otro lado, Walther y D'Addario (2001) no descubrieron diferencias de género en el uso de emoticonos en mensajes de correo electrónico al igual que Huffaker y Calvert ya en el 2005. En cualquier caso, el uso de emoticonos se ha incrementado a lo largo del tiempo como consecuencia de la familiarización con los mismos (Utz, 2000; Markman \& 0shima, 2007).

En los últimos años, la aparición de nuevos emoticonos ha dado lugar a estudios centrados en la comparación de los emoticonos usados en occidente y oriente (conocidos como kaomoji). Pollack (2007) y Yuki, Maddox y Masuda (2007) realizaron unos interesantes estudios en este ámbito de trabajo que mostraron que las diferencias culturales entre ambos grupos de personas se reflejaban en la interpretación que los japoneses daban a estas dos formas de comunicación no verbal en línea.

La investigación en relación a las funciones que desempeñan los emoticonos en las comunicaciones virtuales esgrime dos argumentos que destacan su importancia:

- El primer argumento hace referencia a la longitud de los mensajes. La comunicación con mensajes escritos lleva tiempo, especialmente, cuando se busca claridad. En contraste, la comunicación mediada por ordenador se caracteriza por su rapidez. El uso de emoticonos puede ser la clave para aunar las dos características y conseguir claridad y fluidez. En cualquier caso, esto depende del esfuerzo y de las habilidades comunicativas del usuario. (Baron, 2009).

DOI: ri14.v13i1.716 | ISSN: 1697-8293 | Año 2015 Volumen 13 Nº 1 | ICONO14 
- El segundo argumento se centra en la propiedad que hace del ciberhabla un lenguaje que mezcla características de la escritura y del discurso oral. Si el ciberhabla cuenta con algunas de las características propias del habla, entonces los emoticonos son necesarios para complementar y aclarar el significado de los mensajes. Desde este punto de vista se ve a los emoticonos como un intento de llenar el vacío dejado por la ausencia de elemento paralingüísticos en el ciberhabla (Baron, 2009).

Bajo esta perspectiva se desarrolla el presente trabajo de investigación, cuyos objetivos son ofrecer una visión amplia del uso de emoticonos dentro del lenguaje creado en la red por parte de usuarios de foros de discusión asincrónicos en castellano, así como de las principales funciones que desempeñan.

\section{Metodología}

\subsection{Material y Método}

La metodología elegida para la realización de este estudio ha sido de tipo cualitativo, concretamente el método empleado es el análisis de contenido formal. Se analizan desde punto de vista descriptivo-interpretativo una muestra de mensajes a través de la cual se trata de describir, explicar y comprender el uso y función que desempeñan los emoticonos en el ciberhabla de los usuarios activos de foros virtuales de discusión.

\subsection{Participantes}

\section{Mensajes}

La muestra analizada está formada por 546 mensajes extraídos de foros virtuales de comunicación asincrónica escritos por múltiples, desconocidos y variados autores en diversos momentos temporales. Fueron extraídos al azar y recopilados para su posterior análisis a través de capturas de pantalla de ordenador durante un período de cuatro meses comprendidos entre junio y septiembre de 2013.

ICONO14 | Año 2015 Volumen 13 NN $^{\circ}$ | ISSN: 1697-8293 | DOI: ri14.v13i1.716 
No se conoce la distribución exacta por sexos y edad de los autores de los mensajes debido al uso de "Nicks" que ocultan el género de los usuarios y al hecho de que la mayor parte de foros de discusión cuentan con la opción de ocultar la edad (algunos de ellos sí incluían las edades, no obstante se decidió no recoger estos datos puesto que, al no poder hacerlo en todos los foros elegidos, no se podría emplear este dato en el análisis).

\section{Cuestionario}

El total de personas que participaron en el estudio a través de la realización del cuestionario son un total de 95. El cuestionario estuvo disponible para ser completado en la plataforma digital Google Drive durante los meses de julio y agosto de 2013. Vuelve a desconocerse la distribución exacta por edad y sexo debido al uso de seudónimos.

\subsection{Técnicas de Recogida de Datos}

Las técnicas de recogidas de datos pueden dividirse en dos. La primera técnica empleada consiste en la copia de mensajes virtuales que conforman el diálogo espontáneo y natural creado en torno a distintos temas debatidos en seis foros de discusión asincrónica diferentes. La muestra final está compuesta por un total de 546 mensajes. Todos ellos fueron elegidos al azar y se copiaron utilizando capturas de pantalla para el posterior análisis de los emoticonos que aparecían insertos en ellos.

La segunda técnica de recogida de datos consistió en un cuestionario específicamente construido durante el transcurso de la investigación y que fue aplicado con el objetivo de contrastar y confirmar el significado de algunos de los emoticonos encontrados durante la primera fase de análisis documental de mensajes. A través de él se pretendía contrastar el significado con el que tales símbolos eran utilizados dentro de los mensajes en los cuales aparecían, con su significado fuera del contexto escrito de los mensajes. Los emoticonos elegidos (tabla 1) para formar parte del cuestionario son aquellos cuyo significado parecía más impreciso o ambiguo.

DOI: ri14.v13i1.716 | ISSN: 1697-8293 | Año 2015 Volumen 13 Nº 1 | ICONO14 


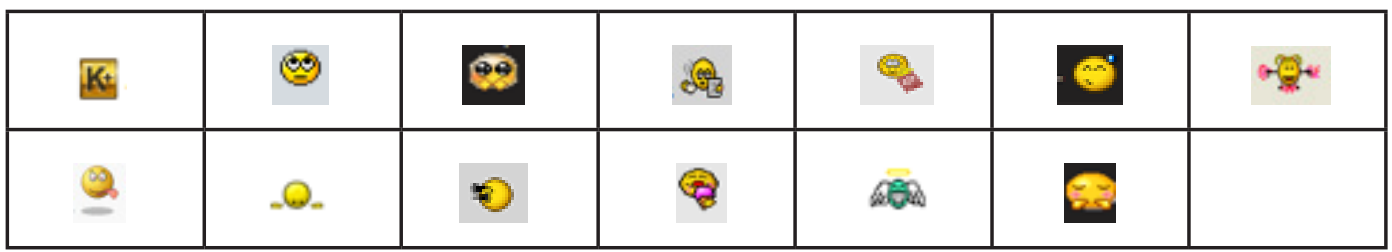

Tabla 1: Emoticonos Cuestionario

La prueba estaba compuesta por un total de 45 ítems divididos en 13 subapartados que se corresponden con cada uno de los 13 emoticonos contrastados. La estructura seguida es siempre la misma:

- ¿Qué significa para ti este emoticón?

- ¿Has utilizado alguna vez este emoticón?

- En el caso de haber respondido "No" en la pregunta anterior, ¿Por qué no lo has utilizado?

\subsection{Procedimiento}

El procedimiento de recogida de información se realizó seleccionando al azar un total de seis foros de discusión asincrónica distintos. El único requisito que se pretendía cumplir con su selección era que cada uno de ellos debía abordar temática distinta. Los mensajes que componen la muestra fueron igualmente seleccionados al azar de entre los numerosos diálogos que de manera natural se habían creado en torno a distintas cuestiones.

Los foros consultados y la distribución de mensajes seleccionados en cada uno de ellos fueron los seis siguientes (tabla 2):

\begin{tabular}{|c|c|c|c|c|c|c|}
\hline $\begin{array}{c}\text { FORO “CAZADORAS } \\
\text { DEL ROMANCE" }\end{array}$ & $\begin{array}{c}\text { FORO “ÁBRETE } \\
\text { LIBRO" }\end{array}$ & $\begin{array}{c}\text { FORO } \\
\text { "COCHES" }\end{array}$ & $\begin{array}{c}\text { FORO } \\
\text { "COCINA" }\end{array}$ & $\begin{array}{c}\text { FORO } \\
\text { “DESDEUNLUGARMEJOR" }\end{array}$ & $\begin{array}{c}\text { FORO } \\
\text { "VOGUE" }\end{array}$ & TOTAL \\
\hline 48 & 179 & 30 & 68 & 86 & 135 & 546 \\
\hline
\end{tabular}

Tabla 2: Distribución de mensajes por foro de discusión

ICONO14 | Año 2015 Volumen 13 N 1 | ISSN: 1697-8293 | DOI: ri14.v13i1.716 
- El foro "Cazadoras del Romance", es un foro de literatura especializado en la novela romántica. La mayor parte de usuarios son mujeres de edades distintas entre sí. Está compuesto por distintos subapartados entre los que destaca un foro dedicado a las presentaciones de los nuevos usuarios, otro dedicado a la novela romántica paranormal, otro a la histórica, otro a la contemporánea y otro más a las novelas románticas fantásticas. Además incluye un foro sobre autores y crítica de libros.

- El foro "Ábrete Libro" es un foro de literatura general. Sus usuarios son tanto hombres como mujeres y las edades son muy heterogéneas entre sí, de modo que pueden encontrarse foreros niños, adultos y ancianos. Se centran en discutir libros de todos los posibles géneros literarios (novelas, poesía, ensayo, biografías, etc.). Se tomaron mensajes de todos los subapartados que conforman el foro.

- El foro "Coches" es un foro que aborda distintos aspectos acerca de los distintos modelos de coches existentes en el mercado. Sus usuarios son de manera mayoritaria hombres que debaten y preguntan cuestiones relacionadas con el tema central del foro.

- El foro "Cocina" es un foro dedicado a compartir recetas de cocina y a preguntar dudas sobre formas de cocinar e ingredientes. Los usuarios son tanto hombres como mujeres y las edades de los participantes son muy distintas entre sí. Se extrajeron mensajes de aquellos foros que en los que se participaba de manera más continuada.

- El foro "Desdeunlugarmejor" es un foro de descargas. Los usuarios de este foro son mujeres y hombres de edades distintas, desde jóvenes hasta adultos y niños. Incluye distintos subapartados: series, películas, música, software, etc. Los mensajes se extrajeron, principalmente de los foros dedicados a descargas de películas y series.

- Por último, el foro "Vogue". Es un foro que está dedicado a tratar diversos temas relacionados con la belleza, la moda, famosos, ocio y cultura, etc. Sus usuarios son de forma mayoritaria mujeres. Los datos extraídos de él fueron tomados de los subapartados de famosos, moda, belleza y ocio y cultura. 


\section{Análisis de datos y Resultados}

\subsection{Análisis general de resultados}

El análisis de la muestra mensajes extraída de foros virtuales de discusión asincrónica ha permitido obtener un total de 135 emoticonos diferentes. Estos emoticonos se usan de manera repetida en los 546 mensajes que componen la muestra objeto de estudio. Para el análisis de datos se establecieron 31 categorías de emoticonos teniendo en cuenta la emoción que transmitían dentro del contexto escrito de los mensajes en los cuales aparecían:

- Alegría, Confusión, No quiero mirar, Tristeza, Emoción, Calor, Sorpresa, Complicidad, Gratitud, Burla, Admiración, Saludo, Vergüenza, Negación, Broma, Afirmación, Amor/Cariño, Silencio, Enfado/Frustración, Bondad, Amenaza, Enfermedad, Duda, Maldad, Acuerdo, Agradabilidad, Sin palabras, Tranquilidad, Interés, Esconderse, Otros.

A primera vista, llama la atención el hecho de encontrar una gran variedad de símbolos, independientemente del tipo de foro y del tipo de usuarios participantes en ellos. Los emoticonos se emplean como herramienta para expresar gran cantidad de emociones y acciones.

La visión general de los mensajes permite observar que los usuarios de foros de comunicación hacen un uso frecuente de distintos tipos de emoticonos. De hecho, todos los mensajes incluían al menos un emoticón, no obstante, es frecuente observar cómo se emplean varios a la vez, en oraciones diferentes o en la misma, con distintos propósitos comunicativos. Este hecho se ha encontrado de manera sistemática en todos los foros consultados, independientemente de la temática tratada y del tipo de usuarios que se aglutinen en torno a ella. Como ejemplo, pueden observarse las figuras 1, 2 y 3. En todos estos mensajes puede observarse cómo el uso de emoticonos amplía las posibilidades comunicativas a través del enriquecimiento de los matices expresivos que se pueden transmitir.

ICONO14 | Año 2015 Volumen $13 N^{\circ} 1$ | ISSN: 1697-8293 | DOI: ri14.v13i1.716 


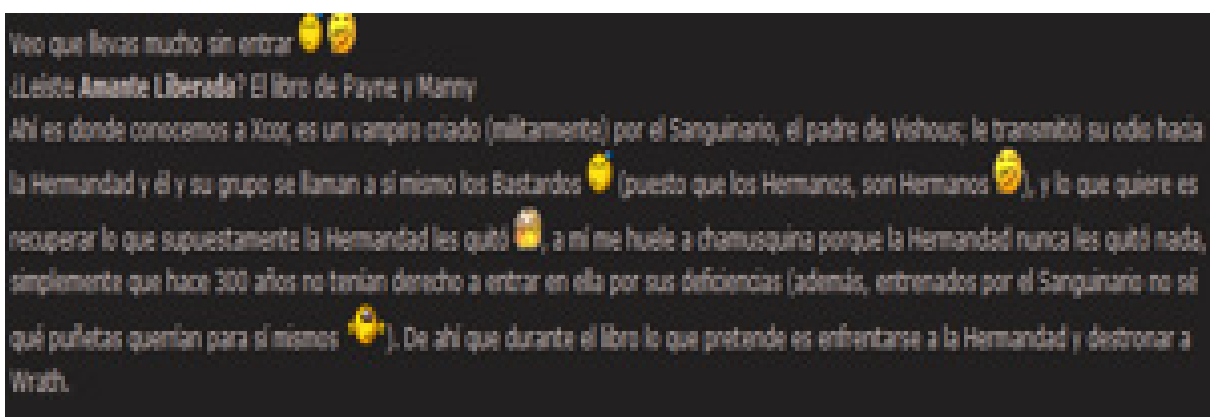

Figura 1: Mensaje ejemplo

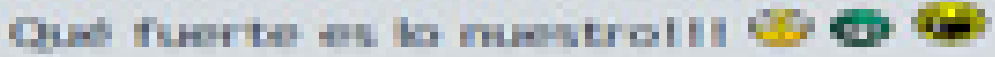

Figura 2: Mensaje ejemplo

\section{M guatar 118 \\ Mis, mis II

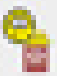

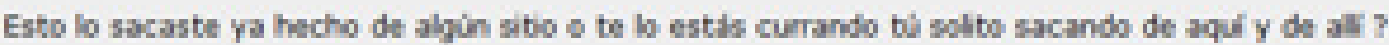

Figura 3: Mensaje ejemplo

Desde otro punto de vista, se puede señalar que se han encontrado muy pocos ejemplos de las estrategias tradicionalmente empleadas por el ciberhabla, con el objetivo de aumentar la expresividad de la comunicación mediada por ordenador. El uso particular de las mayúsculas, el uso exagerado e inapropiado de los signos de puntuación, o la repetición de vocales para enfatizar palabras (Yus, 2011), parece haber caído en desuso con el paso del tiempo, quizás como consecuencia del aumento en la variedad de los emoticonos existentes, que permite al usuario expresar aquello que pretende sin necesidad de acudir a otras estrategias (o haciéndolo en menor medida). Ciertamente se han hallado dentro de la muestra de investigación, algunos mensajes que contienen restos de este tipo de estrategias (concretamente doce), no obstante, su frecuencia, en comparación con el uso de emoticonos, es significativamente menor. Por otro lado, no puede dejar de señalarse que en la mitad de los casos en los que 
estas estrategias fueron encontradas también se recurría al uso del emoticón para aumentar más aún la expresividad de la comunicación. Es posible que solamente a través del uso combinado del emoticón con otras estrategias, puedan satisfacerse las necesidades expresivas de los usuarios de foros de discusión (figuras 4, 5 y 6).

Figura 4: Mensaje ejemplo

La figura 4, es una muestra del uso inapropiado de mayúsculas y de la repetición de letras (en este caso consonantes) para aumentar la expresividad. Este mensaje, tiene la peculiaridad de usar estas estrategias en una palabra onomatopéyica, que por sí sola ya supone un modo de aumentar la expresividad del contenido escrito. Para completar el significado se añade un emoticón, en este caso de sorpresa, que completa el uso que se hace de la estrategia anteriormente citada.

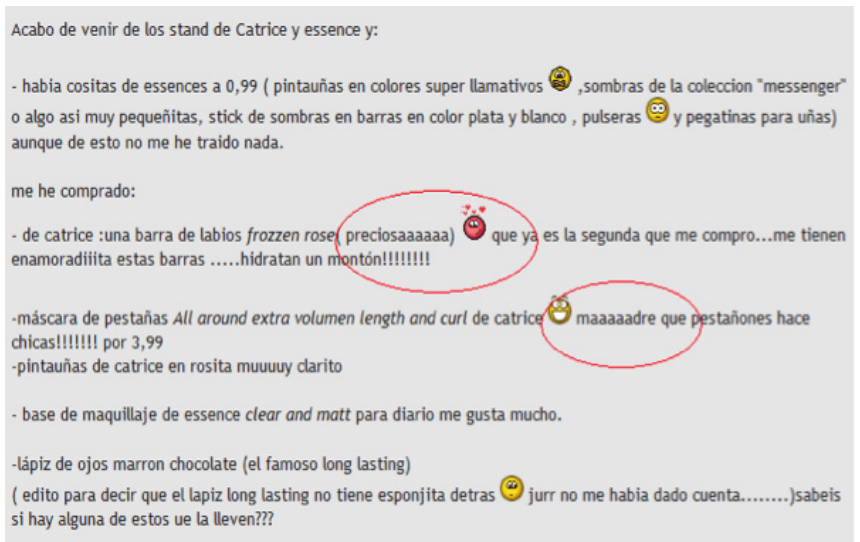

Figura 5: Mensaje ejemplo

La figura 5 ejemplifica el uso que se hace de la repetición de letras (en este caso, vocales) para simular lenguaje hablado (Kalman y Gergle, 2014). Nuevamente el emisor vuelve a utilizar un emoticón al lado de las letras repetidas para complementar el significado emocional que se pretende transmitir. Teniendo en cuenta el contexto escrito se podría decir que el primer emoticón transmite amor, y el segundo asombro. 


\section{Yo no sé si he leído TANTOS libros fantásticos 10 , pero ahora que soy más asidua a este género, iré pensando mi lifta ?}

Figura 6: Mensaje ejemplo

La figura 6 constituye un ejemplo del uso inapropiado de signos de puntuación (en este caso el símbolo de cierre de las oraciones exclamativas). Además se ha añadido un emoticón que completa el tono emocional que se quiere transmitir (alegría).

La siguiente característica encontrada que se puede destacar muestra como los ciberhablantes suelen seleccionar repetidamente un mismo emoticón para magnificar el significado de aquello que se quiere expresar. Es posible que esta estrategia sea una extensión de las ya citadas. Se incluye de manera repetida un emoticón cuando se quiere engrandecer el significado expresivo aportado por el mismo símbolo. Es la misma estrategia usada con la repetición de mayúsculas, signos de puntuación o vocales, pero adaptada al empleo de emoticonos. Los ejemplos de este tipo encontrados en la muestra experimental son bastante numeroso y pueden observarse en aproximadamente un tercio de los mensajes analizados, específicamente, en un total de 186 mensajes diferentes (figura 7).

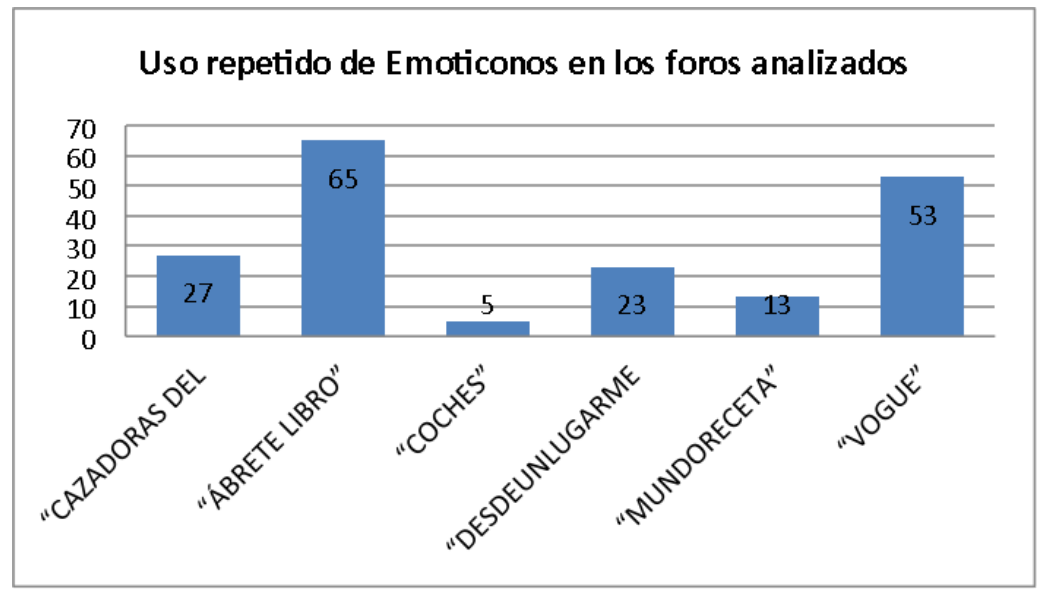

Figura 7 
195 | Isabel Cuadrado, Guadalupe Martín-Mora e Inmaculada Fernández

Algunos ejemplos pueden observarse a continuación (figuras 8, 9 y 10):

Obviamente esta el mueble relleno, sino no te lo hubiera dicho! bohemia.

Figura 8: Mensaje ejemplo

En la figura 8, extraído del foro "Vogue", el autor utiliza dos veces seguidas el mismo emoticón de burla para aumentar la expresividad de la comunicación.

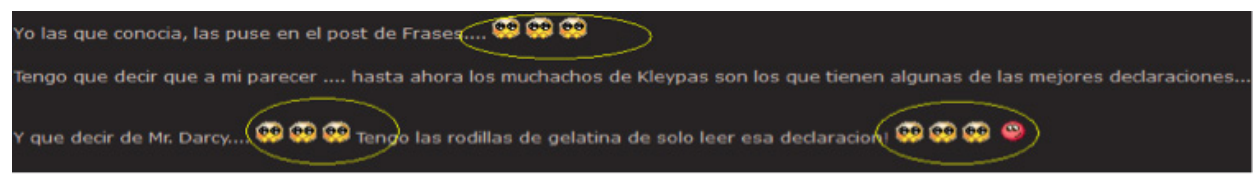

Figura 9: Mensaje ejemplo

El autor del mensaje que puede observarse en la figura 9, extraído del foro "Cazadoras del Romance", utiliza repetidamente al final de tres oraciones seguidas el mismo emoticón. Con él, consigue trasmitir a todos sus compañeros foreros la emoción que le produce el tema debatido.

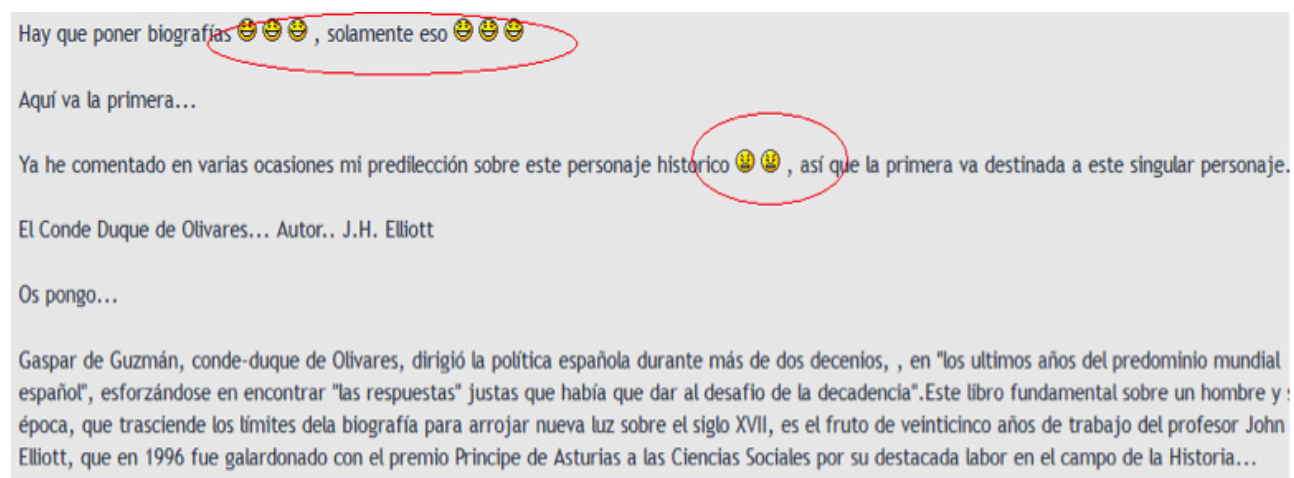

Figura 10: Mensaje ejemplo

La figura 10, extraída del foro "Ábrete libro", muestra la repetición de dos símbolos diferentes que expresan alegría. La sonrisa que muestran los emoticonos da a entender que el autor del mensaje está contento, le gusta el tema tratado y se alegra de poder aportar sugerencias al foro.

ICONO14 | Año 2015 Volumen 13 Nº 1 | ISSN: 1697-8293 | DOI: ri14.v13i1.716 
Es curioso además observar cómo en algunos casos, los emoticonos de los distintos foros analizados son muy similares entre sí y, por tanto, expresarían el mismo significado (tabla 3).

\begin{tabular}{|c|c|c|c|c|c|}
\hline $\begin{array}{c}\text { FORO “CAZADORAS } \\
\text { DEL ROMANCE" }\end{array}$ & $\begin{array}{c}\text { FORO “ÁBRETE } \\
\text { LIBRO" }\end{array}$ & $\begin{array}{c}\text { FORO } \\
\text { "COCHES" }\end{array}$ & $\begin{array}{c}\text { FORO } \\
\text { "COCINA" }\end{array}$ & $\begin{array}{c}\text { FORO } \\
\text { "DESDEUNLUGARMEJOR" }\end{array}$ & FORO “VOGUE" \\
\hline in & $\Theta$ & $\Theta$ & $\Theta$ & $\Theta$ \\
\hline
\end{tabular}

Tabla 3: Ejemplo Emoticonos Similares en distintos foros

Por el contrario, otros solamente aparecen de manera específica en un foro determinado quedando vinculados a una temática concreta, de modo que solo aparecen en ellos, ya que en los demás no tienen ninguna utilidad expresiva. En todos estos casos, los emoticonos constituyen ejemplos específicos del ciberhabla creado en torno al foro. Algunos ejemplos destacables pueden observarse en la tabla 4:

\begin{tabular}{|l|l|}
\hline & $\begin{array}{c}\text { Emoticón Karma (Foro “Desdeunlugarmejor"). } \\
\text { Empleado para dar las gracias a las personas encargadas de colgar los } \\
\text { archivos para ser descargados }\end{array}$ \\
\hline & $\begin{array}{l}\text { Emoticón Qué Bueno (Foro "MundoReceta"). } \\
\text { Empleado para indicar que una receta es apetitosa. }\end{array}$ \\
\hline & $\begin{array}{l}\text { Emoticón Tranquilidad (Foro "Vogue") } \\
\text { Empleado para indicar tranquilidad en la lectura de una noticia }\end{array}$ \\
\hline
\end{tabular}

Tabla 4: Emoticonos ejemplo de Ciberhabla

\subsection{Análisis de las funciones desempeñadas por los emoticonos}

Las principales funciones que se han encontrado y que desempeñarían los emoticonos dentro del contexto lingüístico en la Red, independientemente del tipo de foro son las siguientes: 


\section{Para introducir elementos emotivos en los mensajes.}

Esta es la función más conocida y utilizada por los usuarios. Su uso se remontaría a la invención del primer emoticón y continúa siendo primordial en la actualidad. La muestra analizada aporta numerosos ejemplos que permiten visualizar esta característica. Algunos de ellos son (figura 11, 12 y 13):

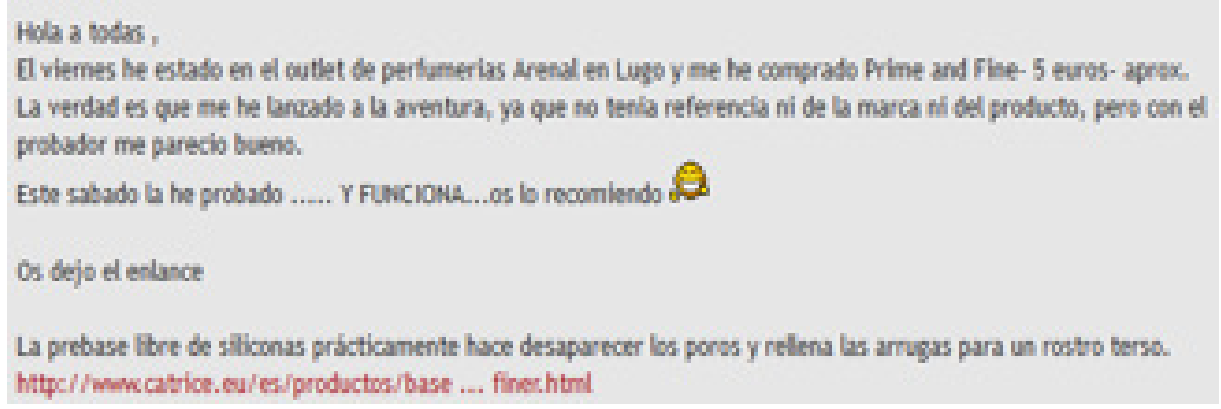

Figura 11: Mensaje ejemplo: Introducción elementos expresivos

En el mensaje de la figura 11, el autor del mensaje expresa su felicidad a través de la incorporación a su escrito de un emoticón que simula un baile de alegría (en el foro del que se ha extraído el mensaje, el emoticón realmente se mueve y baila). Las palabras por sí solas no tienen la capacidad de transmitir la alegría que siente, el emoticón introduce el elemento expresivo que falta.

\section{Que pinta tiene, madd 9 vamos a ver si es lo que parece, mutas gracias, guapa 696}

Figura 12: Mensaje ejemplo: Elementos expresivos

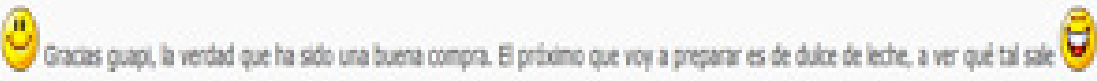

Figura 13: Mensaje ejemplo: Elementos expresivos

Los ejemplo que aparecen en las figuras 12 y 13 son aún más característicos. El emisor utiliza dos emoticonos diferentes en cada mensaje. En el primero, se transmite, a través de un emoticono con ojos en forma de corazón, amor a primera vista. Además, se expresa agradecimiento y cariño con una carita que simula dar un beso 
a la persona receptora de la comunicación. El otro mensaje transmite la alegría que el autor del mensaje siente, y refuerza la emoción expresada añadiendo no uno, sino dos emoticonos distintos, pero con significado similar (alegría).

Dentro de esta característica es importante señalar que los emoticonos son utilizados tanto para indicar el estado emocional del escritor del mensaje, como para hacer referencia al interlocutor e incluirlo dentro de la conversación. Con ello, se aumenta la interactividad entre los foreros y se crea una continuidad entre los mensajes. Así se crea el intercambio de turnos propio de las conversaciones cara a cara en la comunicación mediada por ordenador. A modo de ejemplo del uso dual que realizan los emoticonos (expresión de los sentimientos del autor del mensaje e inclusión del receptor en la conversación), puede observarse la figura 14. En el mensaje, el escritor ha utilizado un emoticón para expresar su alegría, pero también consigue incluir con él, la admiración o agradecimiento que siente hacia el forero al que se dirige el mensaje.

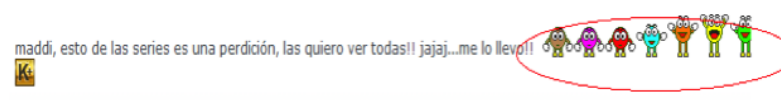

Figura 14: Mensaje ejemplo

\section{Como sustituto de una frase u oración.}

Este segundo uso se caracteriza por la utilización de los emoticonos en sustitución de una frase u oración completa.

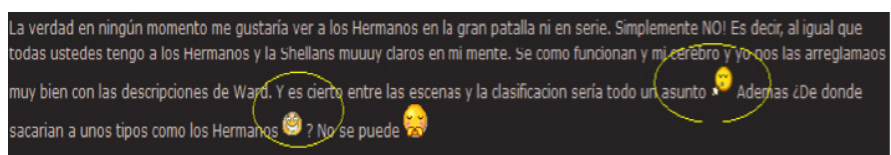

Figura 15: Mensaje ejemplo: Emoticón como sustituto

La lectura de este mensaje ejemplo (figura 15) nos permite observar que se utilizan dos emoticonos como sustituto de palabras. El primero de ellos, permite al autor indicar que el asunto es complicado o cansado, sin necesidad de escribir las palabras necesarias. El receptor cuando lee el mensaje capta el significado, aunque

DOI: ri14.v13i1.716 | ISSN: 1697-8293 | Año 2015 Volumen 13 Nº 1 | ICONO14 
no aparezca escrito de manera explícita. El segundo, muestra que "babea" (le gusta mucho) el tema que se está debatiendo, también sin emplear ninguna palabra. Además se aumenta la expresividad en ambos casos.

\section{Como complemento del mensaje escrito.}

Esta última ocupación desempeñada por los emoticonos se caracteriza por el uso conjunto de texto escrito y emoticonos. Se añaden estos símbolos para completar el significado de aquello que ya está escrito explícitamente con palabras en el mensaje.

Esta función consigue dotar a los mensajes de mayor expresividad. La utilización de emoticonos como complemento del mensaje verbal realiza una función similar a la del lenguaje no verbal en las conversaciones cara a cara. El emisor emplea aspectos como los gestos de las manos y el cuerpo, la posición, el tono de voz, etc. para aumentar la expresividad de aquello que se está diciendo, muchas veces de manera inconsciente. En las conversaciones virtuales, las palabras por sí solas no pueden realizar desempeñar esta función, por tanto se utilizan los emoticonos en un intento de conseguir el mismo resultado expresivo, eso sí, esta vez de manera consciente.

Algunos ejemplos de esta función realizada por emoticonos pueden verse en los mensajes que aparecen en las figuras 16 y 17:

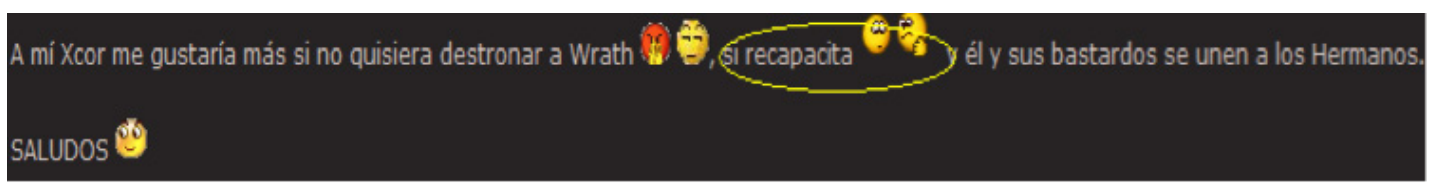

Figura 16: Mensaje ejemplo: Emoticón como complemento del mensaje escrito

El mensaje de la figura 16 muestra una pareja de emoticonos situados al lado de la palabra cuyo significado completan. Ambos emoticonos muestran caras con aspecto pensativo que parecen "recapacitar", que es exactamente el mismo contenido que se expresa con palabras. 
La expresión de las emociones en la Comunicación Virtual: El Ciberhabla | 200

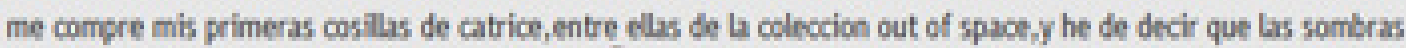
para mi no plgmentan cast nadaque decepion $\theta$ (oonfb en que sea esa coleoton solb)

Figura 17: Mensaje ejemplo: Emoticón como complemento del mensaje escrito

El ejemplo de la figura 17, extraído de otro foro de discusión distinto, utiliza el emoticono exactamente de la misma forma que el anterior. Se incluye un emoticón con aspecto de enfado o decepción justo al lado de las palabras que transmiten verbalmente el mismo significado.

Por último, no puede dejar de hacerse referencia al hecho de que todas las características anteriormente analizadas por separado, suelen encontrarse combinadas dentro de un mismo mensaje. Tal vez con ello se pretenda potenciar al máximo las posibilidades que ofrecen las herramientas que el ciberhabla proporciona para incluir esos aspectos de expresión, emoción y sentimiento que el uso del lenguaje escrito por sí mismo, no permite incluir.

\section{Conclusiones y Discusión de Resultados}

El uso de emoticones en la sociedad de la información se encuentra generalizado dentro del contexto de la comunicación mediada por ordenador. Independientemente de la temática tratada, todos los foros de discusión consultados cuentan con una larga lista de símbolos que pueden ser elegidos en cada uno de los mensajes que se escriben. Este hecho supone una clara muestra del éxito de los mismos. Desde que Falhman inventara en 1982 el primer emoticón, su uso y variedad han crecido de manera espectacular hasta el punto de que, como puede observarse a la luz del análisis de los datos, existen emoticonos para expresar una gran variedad de sentimientos y acciones. Estos resultados parecen concordar con los hallados en 2005 por Huffaker y Calvert, aunque es de esperar que el fenómeno haya crecido aún más hasta llegar al presente.

Desde otro punto de vista, siguiendo a autores como Yus (2001), Crystal (2002), Baron (2009), etc., que afirman que la alfabetización emoticónica no se hace de manera reglada sino sobre la marcha, al mismo tiempo que estos símbolos se uti-

DOI: ri14.v13i1.716 | ISSN: 1697-8293 | Año 2015 Volumen 13 Nº 1 | ICONO14 
lizan para transmitir información en los mensajes, no se ha encontrado en los foros analizados ningún tipo de información adicional que ayude a los usuarios a aprender el uso y función de estos símbolos. Un usuario miembro de un foro concreto aprenderá a emplear los emoticonos más utilizados e interpretará fácilmente el significado que se le da en el contexto espacio-temporal cibernético en el que se mueven. Sin embargo, una persona ajena al foro puede tener problemas a la hora de descifrar el significado de algunos símbolos específicos que no aparecen de manera común en todos los sitios Web que permiten la comunicación virtual. De hecho, este problema ha aparecido en diversas ocasiones a lo largo de esta investigación. Únicamente acudiendo al contexto del mensaje, puede a veces intuirse el significado del emoticón utilizado, $\mathrm{y}$, en otras ocasiones, ni siquiera de este modo se puede estar completamente seguro de interpretar correctamente aquello que el emisor pretendía transmitir. Esto hecho plantea dudas en relación a si los foreros experimentados se encuentran con el mismo problema o, si por el contrario, el proceso de alfabetización emoticónica es tan eficaz que las dudas de interpretación son inexistentes, al menos en el foro en el que participan con asiduidad.

Es obvio que estos símbolos se han convertido en vitales dentro de la comunicación mediada por ordenador. De hecho, su función va más allá de señalar el estado emocional del emisor y se convierten en recursos comunicativos con estatus propio dentro del ciberhabla, tal como señaló Yus (2001) y se ha encontrado ampliamente en el análisis de la muestra objeto de estudio. El uso combinado de todos los recursos que el ciberhabla ofrece muestra que los participantes no adaptan la comunicación cara a cara al entorno virtual, sino que emplean las herramientas que este contexto ofrece para expresar todo aquello que quieren, confirmándose así los resultados que obtuvo Vandergriff en 2013.

Obviamente, escribir es más lento que hablar (Herring, 1999, 2003). La comunicación mediada por ordenador, con la palabra como principal vehículo comunicativo, hereda este problema que afecta directamente al flujo de las comunicaciones virtuales. Algunas estrategias como abreviaturas han sido muy utilizadas en este contexto para dar más elocuencia a las conversaciones (Werry, 1996), y aunque el uso de esta técnica sigue siendo utilizada en nuestros días, el presente estudio resalta el hecho de que los emoticonos parecen emplearse también como un recurso dinamizador.

ICONO14 | Año 2015 Volumen $13 N^{\circ} 1$ | ISSN: 1697-8293 | DOI: ri14.v13i1.716 
Esta función responde probablemente a la característica del ciberhabla señalada por autores como Crystal (2002), y encontrada también en el análisis de datos, que indica que el uso de emoticonos en sustitución de una palabra u oración contribuiría a aumentar la velocidad, continuidad y fluidez de la transmisión de información. En cualquier caso, es importante señalar que esto solamente podría ocurrir cuando el emisor del mensaje está familiarizado con los emoticonos que puede emplear. Un principiante probablemente tardará más en encontrar el emoticón apropiado que sustituya a la frase que quiere escribir, que en escribir todas y cada una de las letras.

En los mensajes, la expresión de emociones se realiza de manera explícita. Con este objetivo parecen emplearse algunas herramientas pragmáticas que sustituyen específicamente a las largas explicaciones escritas acerca del tono emocional del mensaje. El uso de mayúsculas (para "gritar") se encuentra entre estas herramientas ampliamente utilizadas (Varnhaben, McFall, Pugh, Routledge, Sumida-MacDonald \& Kwong, 2009). Sin embargo, el análisis de la muestra apunta a que esta herramienta clásica parece haber sido relegada en favor del uso del emoticón. La evolución en número y variedad de los emoticonos ha hecho que sea más fácil el uso de estos símbolos que el de cualquier otra estrategia. Sorprende además que en algunos foros de discusión analizados se intente evitar el uso de las mayúsculas debido a ser considerado un signo de mala educación, por el que incluso los usuarios llegan a pedir disculpas (figuras 18 y 19).

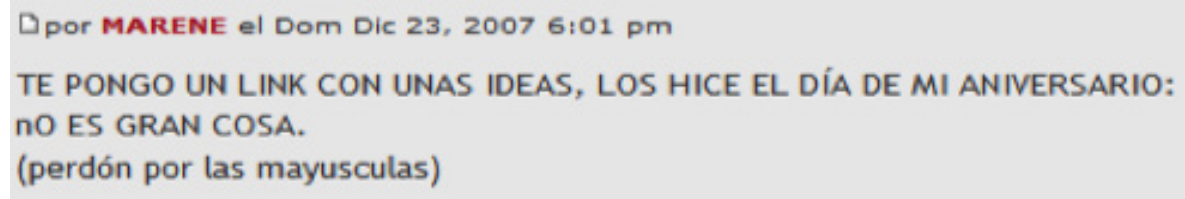

Figura 18: Ejemplo uso de Mayúsculas

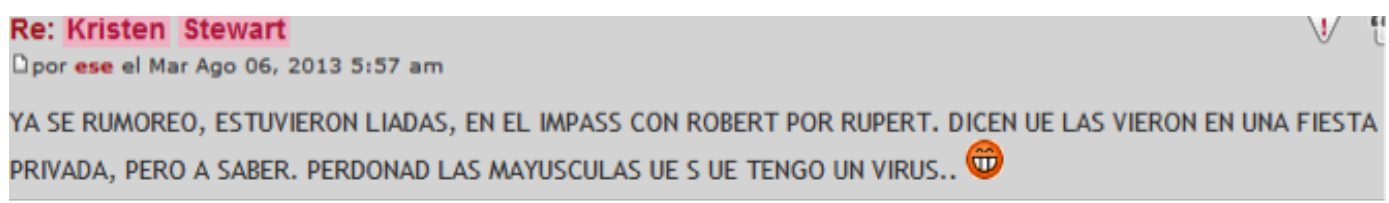

Figura 19: Ejemplo uso de Mayúsculas 
Por otro lado, es importante señalar que, a pesar del importante papel que los emoticonos desempeñan en las conversaciones virtuales, parece que el nivel de expresividad que se quiere transmitir no se consigue con su uso exclusivo. Yus (2001) afirmaba que estos símbolos no consiguen alcanzar el mismo nivel de expresividad que la comunicación no verbal en las conversaciones cara a cara. Como solución a este problema, parece que el paso del tiempo ha hecho que los participantes utilicen una combinación de palabras y emoticonos que se complementan entre sí. El análisis de datos muestra que este fenómeno se encuentra sumamente extendido, es por eso que se clasifica dentro de las principales funciones realizadas por los emoticonos.

Por último, señalar que, si es cierto que el ciberhabla queda constituido como el vehículo comunicativo virtual por excelencia, no hay duda que el emoticón es un importante elemento del mismo que debe ser estudiado de cara a comprender sus implicaciones en el futuro. Obviamente, su uso e interpretación no es algo que se adquiera de manera natural y tampoco es universal, tal y como ocurre con la expresión e interpretación de emociones en el lenguaje no verbal, sin embargo, sería interesante conocer las implicaciones que tendrán en el futuro tanto en el contexto virtual como en el real.

\section{Limitaciones de la investigación}

La mayor limitación del estudio puede situarse en relación al método cualitativo elegido para analizar los datos. Si bien es cierto que el análisis interpretativo-descriptivo de los datos proporciona una caracterización detallada de los mensajes, el uso alternativo de enfoques cuantitativos podría haber supuesto un aumento en la muestra objeto de estudio. Además, el uso de algoritmos informáticos, de manera similar al reciente estudio de Kalman y Gergle (2014), podría haber sido de utilidad en la detección de patrones dentro del ciberhabla. El uso complementario de ambos métodos podría proporcionar una visión mucho más amplia del ciberhabla y de las características que lo forman.

Por otro lado, y puesto que el fenómeno del ciberhabla es extensible a cualquier idioma hablado, se hace necesario la realización de investigaciones en otras lenguas que ayuden a completar el presente estudio.

ICONO14 | Año 2015 Volumen $13 N^{\circ} 1$ | ISSN: 1697-8293 | DOI: ri14.v13i1.716 
La expresión de las emociones en la Comunicación Virtual: El Ciberhabla | 204

\section{Notas}

[1] El gif animado es un formato gráfico utilizado en Internet. Fue creado en 1987 por CompuServer y se caracterizan principalmente por ser imágenes en movimiento (Wikipedia).

\section{Referencias}

Álvarez Martínez, S. (2008). Interacciones sincrónicas escritas en línea y aprendizaje del español: caracterización, perspectivas y limitaciones. Tesis doctoral publicada en línea. Universidad de Lleida, Lleida, España. Recuperado de: http://www. galanet.be/publication/fichiers/Alvarez2008_tesis.pdf

Baron, N.S. (2009). The Myth of Impoverished Signal: Dispelling the Spoken-Language Fallacy for Emoticons in Online Communication. London: Peter Lang.

Byron, K.K. \& Baldridge, D. (2007). E-Mail Recipients' Impressions of Senders' Likability: The Interactive Effect of Nonverbal Cues and Recipients' Personality. Journal of Business Communication, 44(2), 137-160.

Castells, M., Fernández-Ardévol, M., Linchaunqiu, J. \& Sey, A. (2006). Comunicación móvil y sociedad. Una perspectiva global. Barcelona: Ariel-Fundación Telefónica. Cebrián Herreros, M. (2008). La Web 2.0 como Red Social de Comunicación e Información. Estudios sobre el Mensaje Periodístico, 14, 345-361.

Crystal, D. (2001). A linguistic revolution?. Education, Communication and Information, 1(2), 93-7.

Crystal, D. (2002). El lenguaje en la Red. Ediciones Akal.

Crystal, D. (2013). Internet y los cambios en el leguaje. En BBVA (Ed.), C@ mbios. 19 ensayos fundamentales sobre cómo Internet está cambiando nuestras vidas. Recuperado de https://www.bbvaopenmind.com/wpcontent/ uploads/2014/04/BBVA0penMind-libro-Cambio19ensayosfundamentalessobre c\%C3\%B3mointernet-est\%C3\%A1cambiandonuestrasvidasTecnolog\%C3\%ADaIn terentInnovaci\%C3\%B3n.pdf

Darics, E. (2010). Politeness in computer-mediated discourse of a virtual team. Journal of Politeness Research, 6(1), 129--150. doi: 10.1515/jplr.2010.007

ELMUNDO.ES (2007). La sonrisa del ciberespacio, :-), cumple 25 años. Extraído de: http://www.elmundo.es/navegante/2007/09/19/tecnologia/1190164801. html

DOI: ri14.v13i1.716 | ISSN: 1697-8293 | Año 2015 Volumen 13 Nº 1 | ICONO14 
George, E. \& Totschnig, M. (2001). 20 ans de CMO. Dialogue sur l'histoire d'un concept et d'un champ de recherche, en las actas del coloquio La Communication Médiatisée par Ordinateur: un carrefour de problématiques. Universidad de Sherbrook, Quebec, Canadá.

Herring, S. C. (1996). Computer-Mediated Communication: Linguistic, Social, and Cross-Cultural Perspectives. Amsterdam: John Benjamins.

Herring, S. C. (1999). Interactional coherence in CMC. Journal of Computer-Mediated Communication, 4(4). doi: http://dx.doi.org/10.1111/j.1083-6101.1999. tb00106.x

Herring, S. C. (2003). Computer-mediated communication on the internet. In S. B. Barnes (Ed.).

Huffaker, D. A., \& Calvert, A. L. (2005). Gender, identity, and language use in teenage blogs. Journal of Computer-Mediated Communication, 10, 2. doi: http://dx.doi.org/10.1111/j.1083-6101.2005.tb00238.x

Jibril, T.A. \& Abdallah, M.H. (2013). Relevance of Emoticons in Computer-Mediated Communication Contexts: An Overview. Asian Social Science, 19(4), 201-207. doi: http://dx.doi.org/10.5539/ass.v9n4p201

Kalman, Y. M. \& Gergle, D. (2014). Letter repetitions in computer-mediated communication: A unique link between spoken and online language. Computers in Human Behavior, 34, 187-193. doi: http://dx.doi.org/10.1016/j. chb.2014.01.047

Kavanaugh, A., Carroll, J.M., Rosson, M.D., Zin, T.T. \& Reese, D.D. (2005), Community Networks: Where Offline Communities Meet Online. Journal of Computer-Mediated Communication, 10(4). Recuperado de http://jcmc.indiana. edu/vol10/issue4/kavanaugh.html

Lea, M., \& Spears, R. (1992). Paralanguage and Social Perception in ComputerMediated Communication. Journal of Organizational Computing, 2:3(4), 321341.

Markman, K.M., \& Oshima, M.A. (2007). Pragmatic Play? Some Possible Functions of English Emoticons and Japanese Kaomoji in Computer-Mediated Discourse. Trabajo presentado en Association of Internet Researchers Annual Conference 8.0: Let's Play! Vancouver, B.C., Canada.

Markus, M. L (1994). Finding a Happy Medium: Explaining the Negative Effects of Electronic Communication on Social Life at Work. ACM Transactions on

ICONO14 | Año 2015 Volumen 13 N 1 | ISSN: 1697-8293 | DOI: ri14.v13i1.716 
Information Systems, 12(1), 119-149.

Mason, R. (1990). Computer Conferencing in Distance Education. Media and Technology in European Distance Education. A.W. Bates.

Moral Toranzo, F. \& García Loreto, R. (2003). Un nuevo lenguaje en la red. Comunicar, $21,133-136$.

Moral Toranzo, F. (2008). Internet como marco de comunicación e interacción social. Comunicar, 32, 231-237. doi: http://dx.doi.org/10.3916/c32-2009-03005

Moral, F. (2002): Influencia social en comunicación mediada por ordenador. (Tesis doctoral en CD-Rom). Málaga, Universidad de Málaga.

Pollack, A. (1996). Happy in the East ${ }^{\wedge}{ }_{--}{ }^{\wedge}$ or Smiling :-) in the West. New York Times, 12. Recuperado de http://query.nytimes.com/gst/fullpage.html?res=9 905E0D8133EF931A2575BC0A960958260\&n=Top/Reference/Times\%20Topics/ People/P/Pollack,\%20Andrew

Postmes, C., Spears, T R. \& Lea, M. (2000). The Formation of Group Norms in Computer-Mediated Communication. Human Communication Research, 26(3), 341-371. doi: http://dx.doi.org/10.1111/j.1468-2958.2000.tb00761.x

UTZ, S. (2000). Social information processing in MUDs: The development of friendships in virtual worlds. Journal of Online Behavior, 1(1). Recuperado de http://old.behavior.net/J0B/v1n1/utz.html

Valencia Cerino, Y. \& García Martínez, V. (2010). La escritura simbólica y el lenguaje escrito en los usuarios del Messenger. Comunicar, 34, 155-162. doi: http:// dx.doi.org/10.3916/C34-2010-03-15

Vandergriff, I. (2013). Emotive communication online: A contextual analysis of computer-mediated communication (CMC) cues. Journal of Pragmatics, 51, 1-12. doi: http://dx.doi.org/10.1016/j.pragma.2013.02.008

Varnhagen, C.K., Mcfall, G.P., Pugh, N., Routledge, L., Surnida-Macdonald, H. \& Kwong, T.E. (2009). lol: New language and spelling in instant messaging. Reading and Writing, 23, 19-33. doi: http://dx.doi.org/10.1007/s11145-0099181-y

Walther, J.B. \& D'Addario, K.P. (2001). The Impacts of Emoticons on Message Interpretations in Computer-Mediated Communication. Social Science Computer Review, 19, 324-347. doi: http://dx.doi.org/10.1177/089443930101900307 Wei, A. C. Y. (2012). Emoticons and the non-verbal communication: With reference 
to Facebook (Unpublished master's thesis). Department of Media Studies, Christ University, Bangalore - India.

Werry, C.C. (1996). Linguistic and interactional features of internet relay chat. Philadelphia, PA: John Benjamins Publishing.

WIKIPEDIA (http://es.wikipedia.org/wiki/Wikipedia:Portada)

Witmer, D. F. \& Katzman, S. L. (1997). On-line smiles: Does gender make a difference in the use of graphic accents? Journal of Computer-Mediated Communication, 2(4).

Wolf, A. (2000). Emotional expression online: Gender differences in emotion use. Cyberpsychology \& Behavior, 3(5), 827-833. doi: http://dx.doi. org/10.1089/10949310050191809.

Yuki, M., Maddox, M.M. \& Masuda, T. (2007). Are the Windows to the Soul the Same in the East and the West? Cultural Difference in using th

e eyes and mouth as cues to recognize emotions in Japan and the United States. Journal of Experimental Social Psychology, 43, 303-311. doi: http://dx.doi. org/10.1016/j.jesp.2006.02.004

Yus, F. (2001). Ciberpragmática: El uso del lenguaje en Internet. Ariel.

Yus, F. (2011). Cyberpragmatics. Internet-mediated Communication in Context. Benjamins, Amsterdam/Philadelphia. 